\title{
$\mathrm{RCS}$ 모의 데이터를 활용한 기동에 따른 레이다 성능 분석
}

\section{Radar Performance Analysis Based on Target Maneuvering Using Radar Cross Section Simulation Data}

\author{
김현승·전우중 · 박명훈 · 권세웅 · 문현욱 \\ Hyun-Seung Kim $\cdot$ Woo-Joong Jeon $\cdot$ Myung-Hoon Park $\cdot$ Se-Woong Kwon $\cdot$ Hyun-wook Moon
}

요 약

레이다 운용 시 표적의 RCS 변화는 레이다 탐지 성능에 큰 영향을 미친다. 특히, 고기동 표적의 RCS는 급격히 변하므 로 레이다 성능 예측에 있어서 $\mathrm{RCS}$ 예측은 불가피하다. 본 논문에서는 레이다 탐지 성능을 예측하기 위해 M\&S 도구를 개발하였다. 도구는 레이다 모델, 표적 모델 그리고 전파 환경 모델로 구성되어 있고, 기 확보한 RCS 모의 데이터를 활용한 표적 RCS 변화를 반영하였다. 이때 RCS는 레이다와 표적의 상대 자세를 계산하여 RCS 모의 데이터와 이동 평균 방식을 적용한 RCS 가공 데이터로부터 산출하였다. 검증을 위해 두 가지 실측 데이터를 활용하여 레이다 수신 신호를 척도로 모의 결과와 비교하였다. 그 결과, RCS 가공 데이터를 이용한 경우, 다른 RCS 모의 방법을 적용한 결과보다 실측 과 유사함을 확인하였다. 결과적으로 본 연구는 고기동 표적의 RCS 변화에 따른 레이다 탐지 성능 예측에 활용될 것으 로 기대된다.

\begin{abstract}
During radar operation, changes in the radar cross section (RCS) of a target have an adverse effect on the radar detection performance. In particular, the RCS of a target with high maneuvering characteristics can be changed rapidly. Therefore, a precise prediction of the RCS change is essential for satisfactory radar detection performance. In this study, a modeling and simulation (M\&S) tool was developed for the estimation of radar detection performance. The M\&S tool comprises a radar model, target model, and wave propagation model. In particular, pre-obtained RCS simulation data were utilized to consider the effect of the RCS change of the target. Using the developed tool, the RCS based on the relative attitude is extracted from the RCS simulation data and the RCS processed data using the moving average method. The M\&S model is verified by comparing the two test results with the radar received signal power. Consequently, we verified that when using the RCS manufacturing data, the signal power is more similar to the test data than the other RCS simulation methods. It is expected that the radar performance on the high-maneuvering target can be estimated using the developed M\&S tool.
\end{abstract}

Key words: Modeling and Simulation, Radar Cross Section, Prediction of Radar Performance, Moving Average

$$
\text { I. 서 론 } \quad \operatorname{RCS}(\text { radar cross section)는 레이다로부터 방사된 전자기 }
$$

LIG넥스원(주) (LIG NexI Co., Ltd.)

- Manuscript received February 5, 2021 ; Revised March 4, 2021 ; Accepted March 15, 2021. (ID No. 20210205-012)

- Corresponding Author: Hyun-Seung Kim (e-mail: hyunseung.kim2@lignex1.com) 
파가 표적에 입사한 강도 대비 반사되어 다시 레이다로 수신된 강도의 비로 정의할 수 있다. 이러한 RCS 크기는 표적이 정지해 있을 때 외부 형상과 재질에 따라 다르다 ${ }^{[1]}$. 특히 표적이 기동함에 따라 레이다가 바라보는 표적 의 위치가 바뀌므로 RCS 변화량의 차이가 커지게 된다. 특히 스텔스 기능이 탑재되지 않은 전투기의 경우, 레이 다에 포착되는 확률을 줄이고자 고기동을 통해 RCS를 급 변시키고, 이는 탐지 성능에 막대한 영향을 미친다 ${ }^{[2]}$.

따라서 공중전에서 표적의 기동에 따른 RCS 변화 모 의는 레이다 성능 분석을 위해 꼭 확보해야 한다. 이는 전 시 상황에서 RCS 변화에 따른 레이다 탐지 성능을 실시 간으로 예측하는 데 도움을 줄 수 있다. 하지만, 실제 비 행시험을 통해 RCS 변화를 측정하고 분석하는 것은 시간 과 비용 소모가 크기 때문에 $\mathrm{M} \& \mathrm{~S}$ 를 활용한 분석이 필요 하다. 이와 관련해 해상 클러터가 지대한 영향을 미치는 저 RCS 해상 표적에 대한 탐지성능을 분석하고, 생존성 향상을 위한 RCS 범위 분석 연구가 있다 ${ }^{[3],[4]}$. 해상 표적 의 탐지 확률을 증가시키기 위한 신호 모델링을 수행하 였으나, 해상 표적의 RCS는 실제 특성을 반영하지 못한 한계가 존재한다. 또한 다기능 레이다의 공대공 표적 추 적 상황에서 RCS에 따른 탐지확률과 추적 성능을 분석한 연구가 있지만, 여기에서도 RCS는 실측 데이터 기반이 아닌 수식으로 정의하였다 ${ }^{[5]}$. 또한 탄도탄 RCS 변화를 분 석한 연구 ${ }^{[6}$ 에서는 고기동성을 고려하였지만, 탄도탄 비 행경로가 한 가지로 고정된 경우만 분석하였다.

이러한 RCS 특성을 미반영하거나 기동 특성을 반영하 지 못한 기존 연구의 한계를 극복하기 위해 본 논문에서 는 전투기 기종 2 대에 대하여 레이다로부터의 상대 방위 및 고각 1 도 간격에 대한 RCS 모의 데이터를 확보하고, 실제 표적의 기동 시나리오를 바탕으로 기동에 따른 RCS 및 레이다 수신신호 세기 변화를 산출하기 위해 M\&S 도 구를 개발하였다. 나아가 실제 운용환경에서 획득한 실측 레이다 수신신호 세기와 RCS 모의 데이터를 적용한 수신 신호 세기 계산 결과를 비교함으로써 검증 및 분석하였다.

이를 통해 본 연구는 한반도 방공영역에서의 주 표적 기가 고기동하는 상황에서 $\mathrm{RCS}$ 의 실시간적 변화를 산출 하여 레이다의 실시간 탐지 성능 예측 및 설계 최적화에 활용할 수 있을 것으로 기대된다.

\section{II. 모델 구성}

표적으로부터 수신한 신호 세기는 안테나 사양, 운용 환경 손실, 빔 운용 및 표적의 포착 위치에 따른 신호세기 손실, 표적으로부터 반사된 RCS 세기에 따라 실시간으로 변한다. 이러한 운용 특성을 고려한 $\mathrm{M} \& \mathrm{~S}$ 모델은 그림 1 에 도시한 바와 같이 크게 레이다 구동모델, 기동 시나리 오에 따른 표적 운동 모델, 운용환경 모델, RCS 모델, 신 호처리 모델로 구성된다.

여기에서 레이다 구동 모델은 빔 운용을 반영하였고, 표적 운동 모델은 외란이 없는 상황에서 직진, 급선회, 고 도 변경이 가능하다. 지형, 대기 등의 운용환경에 따른 손 실은 APM(advanced propagation model) 모델을 적용하였 다. 표적 RCS 모델은 기 확보한 전투기 2종에 대한 RCS 모의 데이터와 이를 이용한 RCS 가공 데이터를 활용하여 신호처리 모델에서 수신신호 세기를 산출함으로써 탐지 판단에 활용하도록 구성하였다.

최종적으로 표적 기동에 따른 자세 변화와 $\mathrm{RCS}$ 를 통 한 수신 신호세기 모의 결과를 산출하여 실측 결과와 비 교 검증하였다.

\section{2-1 레이다 모델}

본 논문에서 모의하는 레이다 모델은 크게 신호를 송 수신할 때 필요한 안테나 빔 패턴과 구동모델, 표적의 수

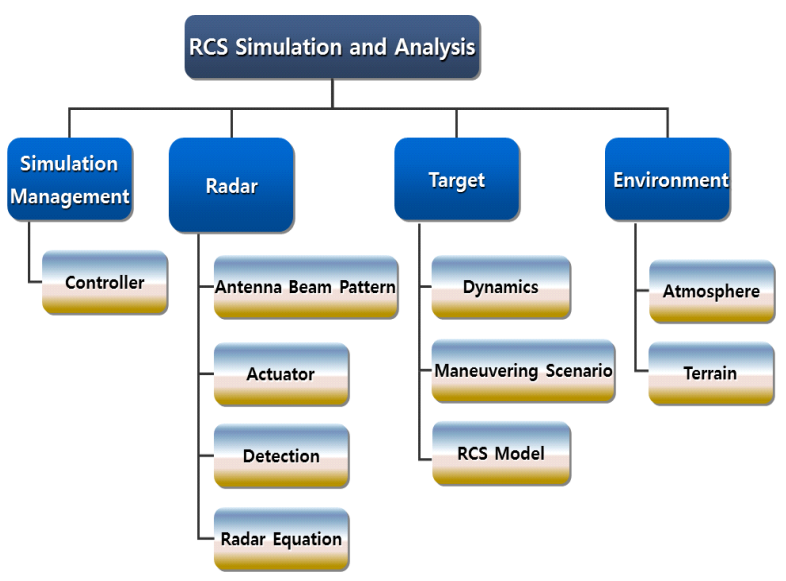

그림 1. M\&S 모델 구성도

Fig. 1. The structure of M\&S model. 
신신호 세기로부터 탐지 여부를 판단하는 순서로 이루어 져 있다.

\section{2-1-1 빔 패턴 모델}

본 논문에서 레이다 송/수신 빔 패턴은 그림 2와 같이 고 각, 방위각에 따른 빔 패턴의 합 패턴으로 표현할 수 있으 며, 빔 조향각에 따른 이득 값을 가지고 방사한다. 빔 패턴 은 $\mathrm{APM}$ 에 반영되어 전파 손실 값을 계산할 때 사용된다.

\section{2-1-2 안테나 구동 모델}

레이다 빔 방사 순서는 설계에 따라 정해져 있고, 일정 속도로 회전한다. 빔별 펄스 수와 PRI(pulse repetition interval)로부터 방사시간을 식 (1)과 같이 계산할 수 있다. 시뮬레이션 시간 간격은 식 (2)를 통해 각도로 환산하여 구동모델에 적용하였다.

$$
\begin{aligned}
& \triangle \text { time }=\text { Pulse }_{\text {num }} \times P R I \\
& \triangle \text { deg }=360^{\circ} \times \Delta \text { time } \times \frac{R P M}{60}
\end{aligned}
$$

\section{2-1-3 탐지판단 모델}

레이다 성능에 RCS 변화의 영향을 분석하기 위해 식 (3)과 같이 표적으로부터 반사된 수신 신호 세기 $\left(P_{R}\right)$ 를 계산하였다. 레이다 설계 변수는 설계치를 적용하고, 표

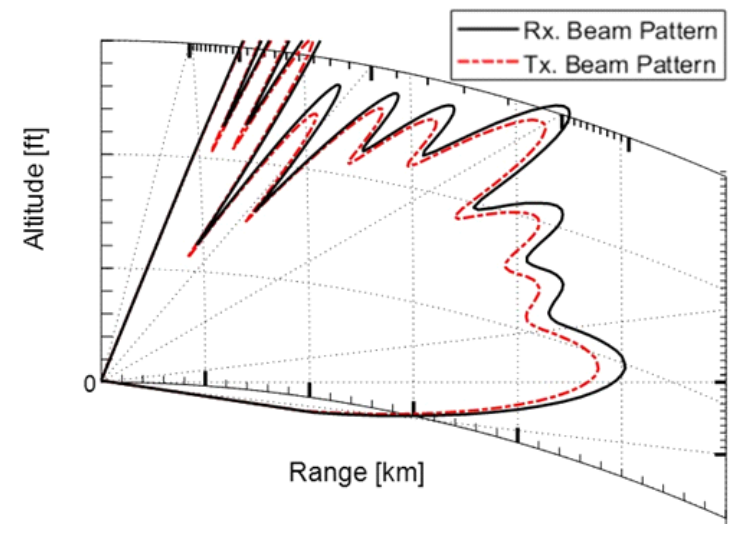

그림 2. 레이다 빔 패턴

Fig. 2. Beam pattern of radar.
적의 위치에 따른 빔 패턴 손실과 운용환경 모델에서 산 출한 전파 손실 $\left(L_{P}\right)$ 을 반영하였다. 계산한 수신신호 세기 와 식 (4)에서 계산한 잡음과 비교를 통해 식 (5)와 같이 수신신호 세기와 잡음의 비가 임계치(threshold)보다 큰 경우, 표적을 탐지한 것으로 판단하였다. 여기에서 임계 치는 정해진 탐지확률 및 오탐지율을 기준으로 하여 설 정하였다 ${ }^{[7]}$.

$$
P_{R}=\frac{P_{t} G_{t} G_{r} \lambda^{2} N \tau \sigma_{\text {target }}}{(4 \pi)^{3} R_{R, T}^{4} L_{p} L_{\text {sys }}}
$$

$P_{t}$ : 송신전력 $(\mathrm{W})$

$\lambda$ : 파장 $(\mathrm{m})$

$G_{t}:$ 송신이득 $(\mathrm{W} / \mathrm{W})$

$G_{r}:$ 수신이득 $(\mathrm{W} / \mathrm{W})$

$N$ : 빔별 펄스 개수

$\tau:$ 펄스 폭 $(\mu \mathrm{s})$

$\sigma_{\text {target }}$ : 표적 $\mathrm{RCS}\left(\mathrm{m}^{2}\right)$

$R_{R, T}$ : 레이다와 표적 간 거리 $(\mathrm{m})$

$L_{p}$ : 대기 손실 $(\mathrm{W} / \mathrm{W})$

$L_{s y s}$ : 시스템손실 $(\mathrm{W} / \mathrm{W})$

$N=k T_{0} B_{n} F_{n}$

$k$ : 볼츠만 상수 $(\mathrm{J} / \mathrm{K})$

$T_{0}$ : 시스템 온도 $(\mathrm{K})$

$B_{n}$ : 잡음 대역폭 $(\mathrm{Hz})$

$F_{n}$ : Noise figure $(\mathrm{W} / \mathrm{W})$

$P_{R}-N>$ Threshold

\section{$2-2$ 표적 모델}

레이다 기준으로 표적의 상대 방위각과 고각의 변화가 $\mathrm{RCS}$ 에 영향을 주기 때문에 표적 운동 모델은 등속 직진 에 급선회, 고도 변경을 추가한 3자유도 운동 모델로 구 성하였다.

\section{2-2-1 표적 운동 모델}

그림 3 은 레이다와 표적 간의 상대 기하를 도시한 것이 다. 레이다를 원점으로 하는 표적의 위치는 동체 좌표계 


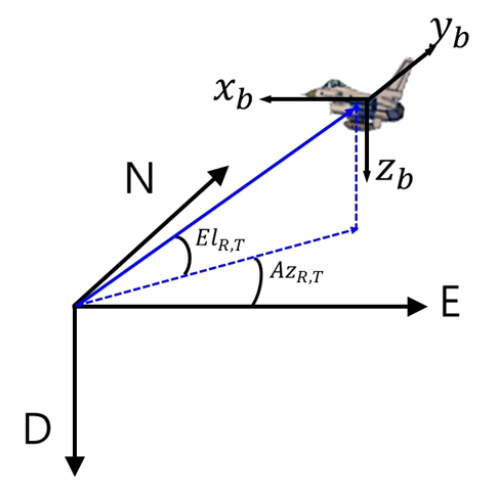

그림 3. $\mathrm{NED}$ 좌표계의 표적 기하학적 구조

Fig. 3. Target geometry in NED coordinate system.

(body-axis)에서 속도, 가속도, 자세 정보를 활용하여 산출 할 수 있다. 이 때 동체 좌표계의 $x$ 축은 표적 기수 방향, $\mathrm{y}$ 축은 우익 방향, $\mathrm{z}$ 축은 지구 중심 방향을 향한다. 식 (6) 의 미분방정식으로부터 동체 좌표계에서의 표적 속도를 산출하고, 식 (7)의 좌표변환을 통해 NED(north-east-down) 좌표계에서의 표적 속도가 나온다. 최종적으로 식 (8)에 서 시뮬레이션 시간 간격(Atime) 이후에서의 표적 위치가 산출된다.

$$
\begin{aligned}
& \dot{V}=a_{x} \quad \dot{\theta}=\frac{a_{z}}{V} \quad \dot{\psi}=\frac{a_{y}}{V \cos \theta} \\
& V_{B}=\left[\begin{array}{c}
V \cos \theta \cos \psi \\
V \sin \psi \\
-V \sin \theta \sin \psi
\end{array}\right] \\
& C_{N}^{B}=R_{1}(\phi) R_{2}(\theta) R_{3}(\psi) \\
& =\left[\begin{array}{ccc}
1 & 0 & 0 \\
0 & \cos \phi & \sin \phi \\
0 & -\sin \phi & \cos \phi
\end{array}\right]\left[\begin{array}{ccc}
\cos \theta & 0 & -\sin \theta \\
0 & 1 & 0 \\
\sin \theta & 0 & \cos \theta
\end{array}\right]\left[\begin{array}{ccc}
\cos \psi & \sin \psi & 0 \\
-\sin \psi & \cos \psi & 0 \\
0 & 0 & 1
\end{array}\right] \\
& V_{N}=C_{B}^{N} V_{B}=\left(C_{N}^{B}\right)^{T} V_{B} \\
& {\left[\begin{array}{c}
N_{T} \\
E_{T} \\
D_{T}
\end{array}\right]=V_{N} \Delta \text { time }}
\end{aligned}
$$

$V:$ 기수 방향 비행속도 $(\mathrm{m} / \mathrm{s})$

$\phi:$ 표적 롤 각 $(\mathrm{deg})$

$\theta$ : 표적 피치 각 $(\mathrm{deg})$

$\psi$ : 표적 요 각 $(\mathrm{deg})$

$\left[a_{x}, a_{y}, a_{z}\right]:$ 가속도 명령 $\left(\mathrm{m} / \mathrm{s}^{2}\right)$
$\left[N_{T}, E_{T}, D_{T}\right]$ : 표적 $\mathrm{NED}$ 좌표계 위치 $(\mathrm{m})$

가속도 명령은 표적이 선회할 때 선회반경과 비행 속 도에 의해 식 (9)와 같이 생성할 수 있다.

$$
a_{y}=\frac{V^{2}}{R_{\text {turn }}}=g \sqrt{\left(n^{2}-1\right)}
$$

$R_{\text {turn }}$ : 선회반경 (m)

$g:$ 중력 가속도 $\left(\mathrm{m} / \mathrm{s}^{2}\right)$

$n$ : 하중배수

\section{2-2-2 레이다와 표적 간 상대 자세}

그림 3에서 레이다가 표적을 바라보는 상대 위치로부터 상대 방위각 및 고각을 식 (10) 식 (12)로 산출할 수 있다. 여기서 레이다는 첨자 $R$, 표적은 첨자 $T$ 로 표기한다.

$$
\begin{aligned}
& P_{r e l}=\left[N_{R} E_{R} D_{R}\right]-\left[N_{T} E_{T} D_{T}\right] \\
& P=C_{N}^{B} P_{r e l} \\
& A z_{R, T}=360^{\circ}-\tan ^{-1}\left(\frac{P_{y}}{P_{x}}\right) \\
& E l_{R, T}=\tan ^{-1}\left(\frac{-P_{z}}{\sqrt{P_{x}^{2}+P_{y}^{2}}}\right)
\end{aligned}
$$

$P_{r e l}$ : 레이다와 표적의 $\mathrm{NED}$ 상대 위치 (m)

$P$ : 레이다와 표적의 동체좌표계 상대 위치 $(\mathrm{m})$

$A z_{R, T}$ : 상대 방위각 $(\mathrm{deg})$

$E l_{R, T}$ : 상대 고각 $(\mathrm{deg})$

\section{2-2-3 기동 시나리오 모델}

표적 기동 시나리오 모델은 표적의 초기 운동 파라미 터인 위치, 속도, 가속도를 입력 받아 운동 모델이 모의될 수 있도록 통제한다. 본 논문에서 모의하는 표적 기동 시 나리오는 실제 시험을 바탕으로 등속 직진 및 선회, 고도 변경으로 설정하였다.

\section{2-3 운용환경 모델}

본 논문에서는 실제 레이다가 운용되는 사이트에서의 
지형 및 대기 환경을 고려한 전파 특성을 계산하기 위해 서 APM 모델을 적용한 상용 프로그램인 AREPS(advanced refractive effects prediction system)를 사용하였다. 이를 통 해 레이다 설치 위치와 대기상태 및 지형 정보, 레이다 성 능 제원 등에 따른 전 방위에 대한 고각 별 손실 값을 획 득하여 레이다 탐지판단 시 전파 손실 값 $\left(L_{P}\right)$ 으로 적용하 였다 ${ }^{[8]}$.

\section{2-4 이동평균 방식을 적용한 표적 RCS 모델}

표적기 2종에 대해 기 확보한 RCS 모의 데이터는 그림 4(a)와 같고, 좌측은 RCS 대푯값이 $2 \mathrm{~m}^{2}$, 우측은 RCS 대 푯값이 $3 \mathrm{~m}^{2}$ 이다. 모의 데이터는 표적기 2종에 대한 모형 을 생성하여 CFD(computational fluid dynamics) 방법으로 획득하였다 ${ }^{[9],[10]}$. 표적 기수를 중심으로 우익 방향으로 방 위각이 1 도씩 증가한다. 실선 동심원은 $20 \mathrm{dBsm}$ 간격이 고, 점선 한 칸은 $5 \mathrm{dBsm}$ 씩 변한다.

그림 4(a)에서 RCS 모의 데이터의 방위각 해상도가 1 도로 크기 때문에 각도 변화에 따른 $\mathrm{RCS}$ 가 최대 $25 \mathrm{~dB}$ 의

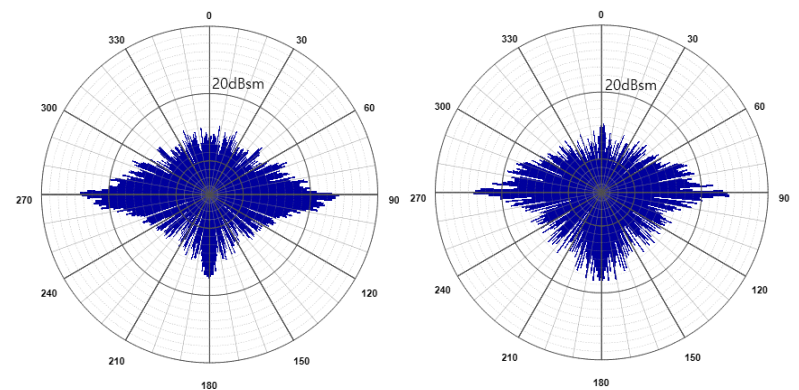

(a) RCS raw data (target-1/target-2)

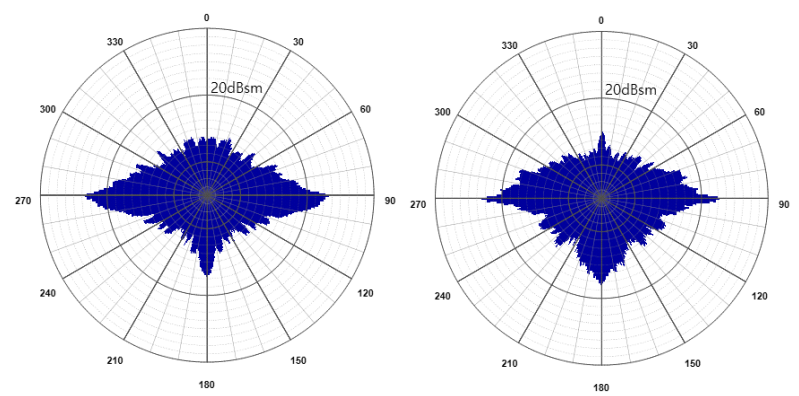

(b) RCS processed data (target-1/target-2)

그림 4. 표적 1 및 2의 RCS 데이터

Fig. 4. RCS data for target 1 and target 2.
표 1. 방위에 따른 RCS

Table 1. RCS according to azimuth.

\begin{tabular}{|c|c|c|c|}
\hline \multicolumn{2}{|c|}{ Target } & Average $\left(\mathrm{m}^{2}\right)$ & Std $(\mathrm{dB})$ \\
\hline \multirow{2}{*}{1} & Raw data & 2.08 & 5.19 \\
\cline { 2 - 4 } & Processed data & 2.07 & 1.14 \\
\hline \multirow{2}{*}{2} & Raw data & 2.92 & 5.63 \\
\cline { 2 - 4 } & Processed data & 2.91 & 1.24 \\
\hline
\end{tabular}

큰 폭으로 변하는 것을 확인할 수 있다. 반면 표적의 상대 자세는 연속적으로 변하기 때문에 방위각 해상도로 인한 데이터 민감성 문제가 발생한다. 이러한 왜곡을 보정하기 위해 이동평균법을 적용한 RCS 가공 데이터를 생성하였 다. 결과는 그림 $4(\mathrm{~b})$ 와 같고 ${ }^{[1]}$, 가공처리는 3 도 간격의 이동평균 방식을 적용하여 RCS 변화 폭을 최대 $5 \mathrm{~dB}$ 이 내로 완화시켰다.

표 1 과 같이 RCS 평균값은 대푯값과 거의 유사하며, 기 확보한 RCS 모의 데이터(raw data)에 비해 RCS 가공 데이터(processed data)는 표적 1의 경우, 표준편차가 5.19 $\mathrm{dB}$ 에서 $1.14 \mathrm{~dB}$ 로, 표적 2의 경우, $5.63 \mathrm{~dB}$ 에서 $1.24 \mathrm{~dB}$ 로 변동 폭이 줄어듦에 따라 수신신호 세기의 예민도를 낮 추었다.

결과적으로 식 (6) 식 (9)로부터 계산된 표적 위치를 이용하여 식 (12)에서 레이다와 표적의 상대자세를 산출 하고, 그림 4에서 자세에 해당하는 RCS를 호출하여 식 (3)의 레이다 방정식으로 수신신호 세기를 구할 수 있다.

\section{M\&S 결과 및 성능 검증}

\section{3-1 시나리오}

본 논문에서는 2-4에서 정의한 표적기 2종에 대해 기 확보한 RCS 모의 데이터와 동일한 기종에 대해 실측 결 과를 활용하여 모의 결과와 비교 및 분석하여 검증하였 다. 그림 5는 RCS 실측데이터를 확보한 시험에 대한 표 적 기동 시나리오를 도시한 것으로 레이다는 한반도 내 특정 지점에 고정되어 있다. 시나리오 $\mathrm{A}$ 는 레이다를 기 준으로 북동 방향으로 퇴각하는 기동이고, 시나리오 $\mathrm{B}$ 는 남서 방향으로 퇴각하는 비행 궤적을 가지며, 표 2와 같 이 초기 운동 파라미터를 설정하였다. 


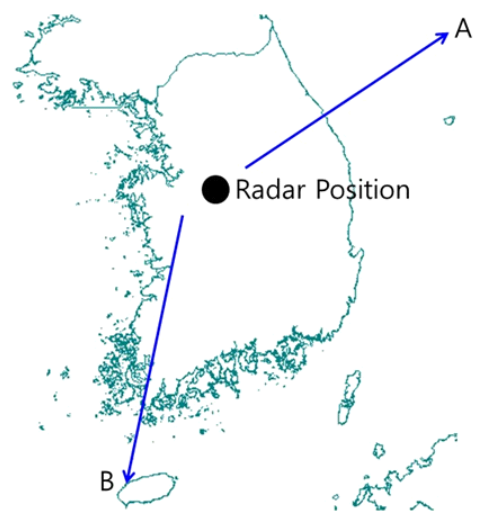

그림 5. $\mathrm{M} \& \mathrm{~S}$ 모델 분석 시나리오 개념도

Fig. 5. The concept of M\&S model analysis scenario.

표 2. $\mathrm{M} \& \mathrm{~S}$ 모델 시나리오 요약

Table 2. Summary of M\&S model scenario.

\begin{tabular}{|c|c|c|}
\hline \multirow{2}{*}{ Item } & \multicolumn{2}{|c|}{ Scenario } \\
\cline { 2 - 3 } & A & B \\
\hline Altitude [ft] & 30,000 & 20,000 \\
\hline Velocity [m/s] & 400 & 370 \\
\hline Azimuth [deg] & $\begin{array}{c}\text { Northeast } \\
(65)\end{array}$ & $\begin{array}{c}\text { Southwest } \\
(190 \sim 230)\end{array}$ \\
\hline Atmosphere & Standard & Standard \\
\hline Target type & 1 (RCS: $\left.2 \mathrm{~m}^{2}\right)$ & 2 (RCS: $\left.3 \mathrm{~m}^{2}\right)$ \\
\hline
\end{tabular}

운용 중인 레이다 빔 패턴, 설계 변수를 반영하고, 전파 환경 모델은 레이다 사이트의 DTED(digital terrain elevation data) 정보와 시험 당일 기상 관측 데이터를 활용하여 $\mathrm{APM}$ 모델로부터 전파 손실 값을 산출하였다. 모의 시 2-2-1 표적 운동모델로 표적 기동을 모의하고, 식 (12)를 이용하여 상대자세를 산출하였다. 표적 RCS는 크게 3가 지로 1) 대푯값으로 고정한 값, 2) 기 확보한 RCS 모의 데 이터, 3) RCS 가공 데이터(이동평균 방식)로 나누어 실측 과 비교·분석하였다.

\section{3-2 M\&S 분석 결과}

그림 6, 그림 7은 시나리오 $\mathrm{A}, \mathrm{B}$ 에 대하여 레이다 회전 수에 따른 레이다와 표적 간의 상대자세 변화를 나타낸

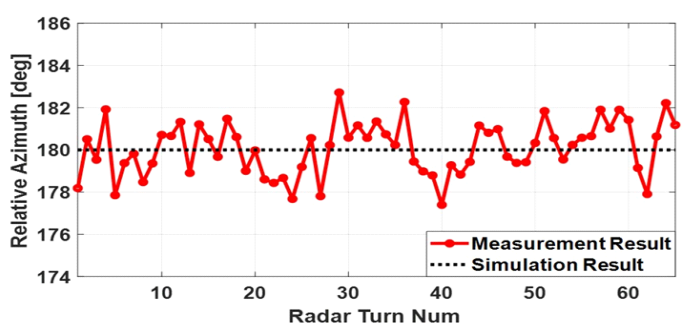

(a) 상대 방위각 변화

(a) Relative azimuth angle change

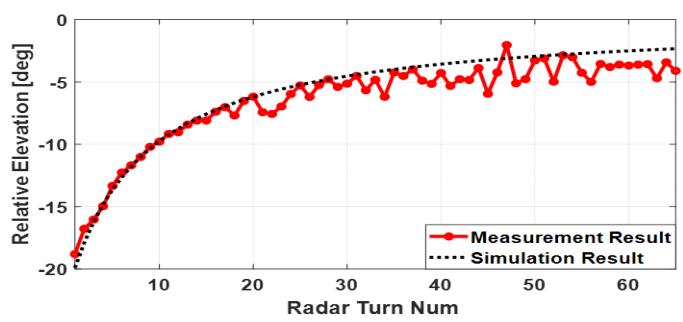

(b) 상대 고각 변화

(b) Relative elevation angle change

그림 6. 상대 자세 변화 (시나리오 A)

Fig. 6. Relative attitude change (scenario A).

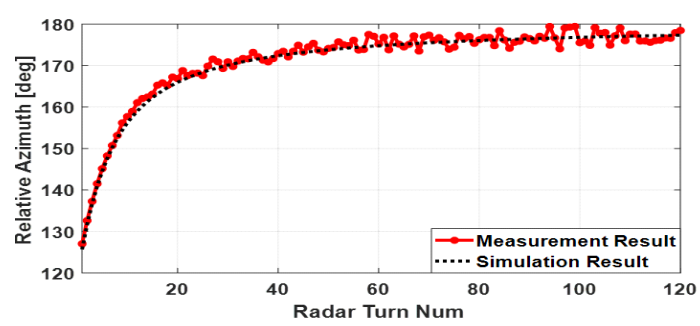

(a) 상대 방위각 변화

(a) Relative azimuth angle change

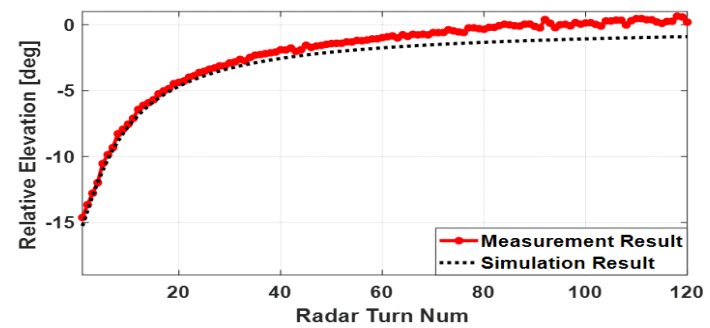

(b) 상대 고각 변화

(b) Relative elevation angle change

그림 7. 상대 자세 변화 (시나리오 $\mathrm{B}$ )

Fig. 7. Relative attitude change (scenario B). 
것이다. 여기에서 실측 데이터의 경우, 레이다가 표적을 탐지한 경우에만 값이 저장된다. 모의 시 표적 기동에 외 란이 없다고 가정하였기 때문에 실측과의 차이가 존재하 지만, 표 3과 같이 오차 편차가 2도 이내임을 확인하였다. 시나리오 $\mathrm{A}$ 의 경우에는 고정된 레이다로부터 표적이 멀어지는 방향으로 기동하기 때문에 방위각이 180 도로 일정하며, 고각에 따른 RCS 변화만을 분석할 수 있다. 이 는 고각 방향에 대한 실측 데이터와 RCS 모의 데이터의 유사성 검증을 용이하게 하며, RCS 가공 데이터의 신뢰 성 확보에 이용하였다. 시나리오 $\mathrm{B}$ 의 경우에는 고정된 레 이다 위치에 대해 표적이 멀어짐에 따라 방위각이 130 도 에서 180 도로 수렴하며, 고각이 변한다.

그림 8 , 그림 9는 시나리오 $\mathrm{A}, \mathrm{B}$ 에 대해 레이다 회전수 에 따른 레이다 수신신호의 실측 및 모의 결과를 나타낸 것이다. 그림 $8(\mathrm{a})$ 과 그림 $9(\mathrm{a})$ 는 레이다 회전수에 따른 실 측 데이터, RCS 대푯값, $\mathrm{RCS}$ 모의데이터, RCS 가공데이 터(이동평균방식)에 대한 모의 탐지성능을 도시한 것으로 두 가지 시나리오에 대해 모두 3) RCS 가공 데이터를 적 용한 결과가 실측과 경향이 가장 유사하며, 오차가 작은 것을 확인할 수 있다. 이 때, 실측 데이터는 레이다 수신신 호 세기로 획득되는 값이고, 역으로 RCS를 산출할 수 있 지만, 환경적인 요인 등에 의해 오차가 발생할 수 있다. 따 라서 실측 결과에 모의를 맞추고자 RCS를 전처리 과정을 통해 변화를 모의하고, 이로부터 식 (3)에서의 수신신호 세기를 산출하여 비교함으로써 명확한 분석을 하였다.

특히 시나리오 $\mathrm{A}$ 에서 방위각이 일정한 기동 상황에서 $\mathrm{RCS}$ 를 대푯값으로 고정한 경우, 실측 결과와 차이가 10 $\mathrm{dB}$ 이상이고 경향이 유사하지 않기 때문에, 고각에 따른 $\mathrm{RCS}$ 변화가 지대한 영향을 보임을 알 수 있다. 또한 RCS

표 3. 실측 및 시뮬레이션 결과 오차

Table 3. Error between measurement and simulation result.

\begin{tabular}{|c|c|c|c|}
\hline \multirow{2}{*}{ Scenario } & \multicolumn{2}{c|}{ Error (measurement-simulation) [deg] } \\
\cline { 3 - 4 } & Azimuth & 0.25 & Std \\
\hline \multirow{2}{*}{ A } & Average & 1.93 \\
\cline { 2 - 4 } & Elevation & 0.83 & 0.96 \\
\hline \multirow{2}{*}{ B } & Azimuth & 0.04 & 1.24 \\
\cline { 2 - 4 } & Elevation & 0.63 & 0.44 \\
\hline
\end{tabular}

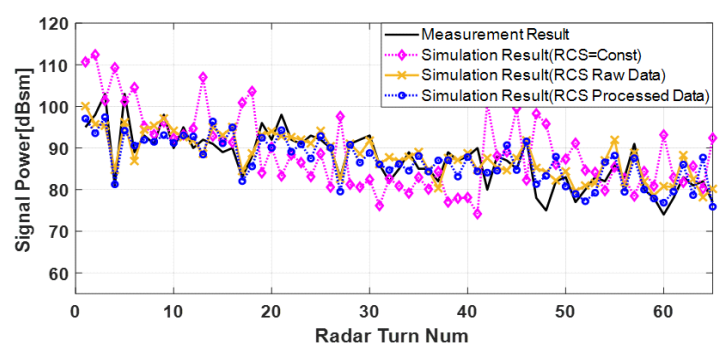

(a) 실측 및 모의 탐지 성능

(a) Detection performance of test and simulation

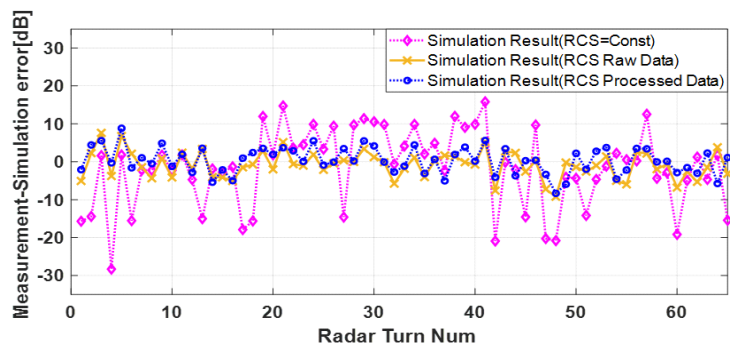

(b) 오차 (실측 - 각각의 모의 결과)

(b) Error (measurement-each simulation)

그림 8. 탐지 성능 결과 (시나리오 $\mathrm{A}$ )

Fig. 8. Result of detection performance (scenario A).

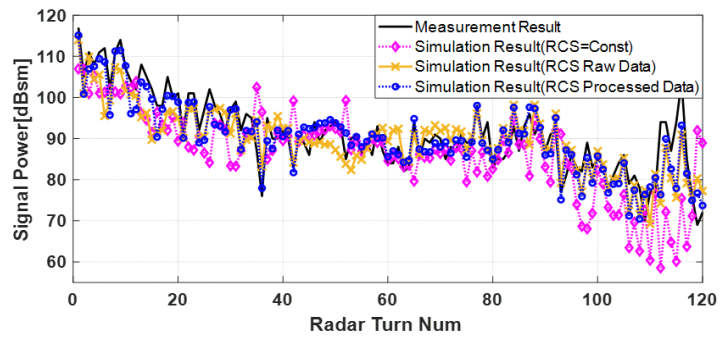

(a) 실측 및 모의 탐지 성능

(a) Detection performance of test and simulation

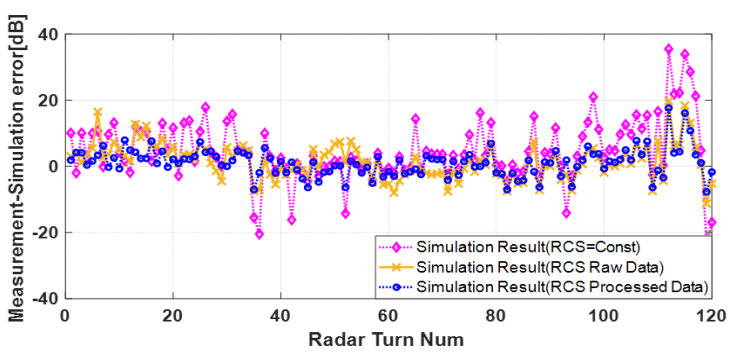

(b) 오차 (실측 - 각각의 모의 결과)

(b) Error (measurement-each simulation)

그림 9. 탐지 성능 결과 (시나리오 B)

Fig. 9. Result of detection performance (scenario B). 
THE JOURNAL OF KOREAN INSTITUTE OF ELECTROMAGNETIC ENGINEERING AND SCIENCE. vol. 32, no. 5, May. 2021.

모의 데이터와 이동평균방식을 적용한 RCS 가공 데이터 를 적용한 모의 결과는 거의 유사하지만, 레이다 회전수 가 증가하여 고각이 0 도에 가까워질수록 가공 데이터를 적용한 결과가 실측과 유사함을 검증하였다.

그림 8(b)와 그림 9(b)는 시나리오 A, B에 대해 그림 8(a)와 그림 9(a)에 도시한 실측과 모의 수신신호 세기의 오차를 나타낸 것이다. 두 시나리오에 대해 RCS를 고정 값으로 적용한 모의 결과가 RCS 모의/가공 데이터를 적 용한 경우보다 실측과의 오차 수준이 큰 것을 확인할 수 있다. 시나리오 B의 경우, 레이다 회전수가 100 120회일 때 실제 레이다 운용 사이트로부터 해당하는 거리에 지 형 및 대기에 따른 영향이 크게 나타나는 것을 확인했다. 특히 방위각과 고각이 거의 일정하게 유지되어 RCS 값이 $10 \mathrm{dBsm}$ 으로 유지되는 구간이므로 외부 환경의 영향으 로 수신신호 세기가 급증하며, 이는 모의 결과에서도 확 인할 수 있었다.

표 4는 실측과 모의 결과의 오차에 대한 평균 및 표준 편차를 나타낸 것이다. RCS를 대푯값으로 고정했을 때 실제 표적 기동에 따른 영향성을 반영하지 못하기 때문 에 시나리오 $\mathrm{A}$ 에서 고각방향으로 편차 $10 \mathrm{~dB}$ 이상이 발 생한다. 또한 시나리오 $\mathrm{B}$ 에서 고각, 방위각이 변함에 따 라 유사한 오차 수준을 보인다. 두 가지 시나리오에 대해 $\mathrm{RCS}$ 모의 데이터를 적용한 모의 결과는 RCS 가공 데이 터를 적용했을 경우보다 실측과의 오차 평균은 $0.5 \mathrm{~dB}$ 크 고, 편차는 유사함을 확인하였다. 이는 RCS 모의 데이터 의 방위각 및 고각 1 도에 대한 값 변동이 크기 때문에 $\mathrm{RCS}$ 가공 데이터를 적용해야 RCS 값 자체가 실측과 유

표 4. 실측 및 시뮬레이션 결과 오차

Table 4. Error between measurement and simulation result.

\begin{tabular}{|c|c|c|c|}
\hline \multicolumn{2}{|c|}{ Scenario } & \multicolumn{2}{c|}{ Error [dB] } \\
\cline { 3 - 4 } \multicolumn{2}{|c|}{ A } & Average & Std \\
\hline \multirow{4}{*}{ B } & RCS constant & 1.73 & 10.32 \\
\cline { 2 - 4 } & Raw data & 0.84 & 3.51 \\
\cline { 2 - 4 } & Processed data & 0.36 & 3.50 \\
\hline \multirow{3}{*}{} & RCS constant & 4.59 & 9.43 \\
\cline { 2 - 4 } & Raw data & 1.49 & 5.47 \\
\cline { 2 - 4 } & Processed data & 1.01 & 4.23 \\
\hline
\end{tabular}

사해져 오차 수준이 줄어드는 것을 의미한다.

표적 기동에 따라 레이다 회전수에 대한 RCS 변화는 그림 10에 도시하였다. 그림 10(a)에서 볼 수 있듯이 레이 다로부터 표적이 꼬리날개만 보이는 경우, 방위각이 180 도로 고정되기 때문에 고각방향으로 RCS 모의 데이터와 $\mathrm{RCS}$ 가공 데이터 간의 큰 차이가 없다. 고각이 -20 도에 서 -10 도인 레이다 회전수 $0 \sim 10$ 회 구간에서는 RCS 모 의 데이터의 변동 폭이 크지만 경향은 유사하다. 그림 10(b)에서 방위각 160 170도 근방인 레이다 회전수 20 60 회 구간에서는 표적 주익의 영향이 큰 영역이므로 그 림 4(a)에서 볼 수 있듯이 RCS 모의 데이터의 변동이 다 른 각도에 비해 $10 \mathrm{~dB}$ 이상으로 매우 크기 때문에 RCS 가공 데이터를 적용해야 실측과 결과가 유사하다.

이러한 결과를 통해 레이다 성능 분석 시 표적 RCS 모 의/가공 데이터를 M\&S 도구에 적용함으로써 실측과의 비교를 통해 검증하고 최적화된 레이다 수신모델을 검증 하였다. 레이다 체계 설계 시 운용환경 외에 표적 기동에

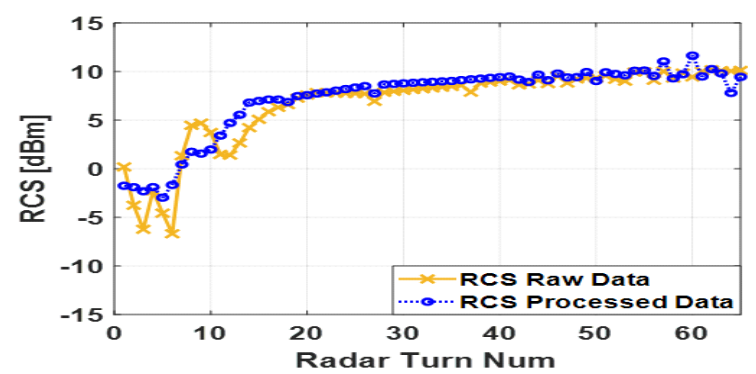

(a) 표적 기동에 따른 $\mathrm{RCS}$ 변화 (시나리오 $\mathrm{A}$ )

(a) RCS change during target maneuvering (scenario A)

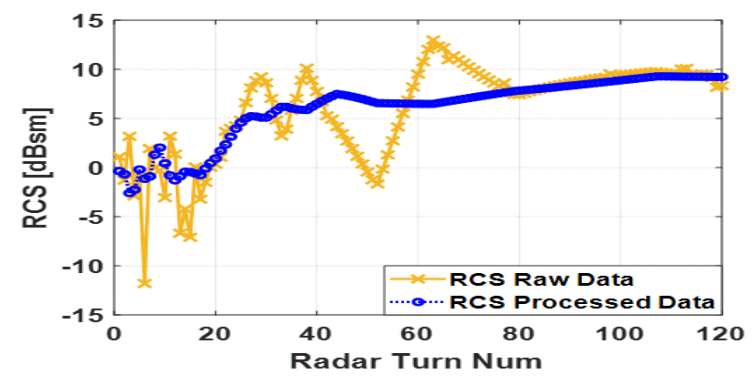

(b) 표적 기동에 따른 $\mathrm{RCS}$ 변화 (시나리오 $\mathrm{B}$ )

(b) RCS change during target maneuvering (scenario B)

그림 10. 기존 데이터 및 가공 데이터 RCS

Fig. 10. RCS between raw data and processed data. 
따른 $\mathrm{RCS}$ 변화를 구체적으로 모사할 수 있음에 따라 레 이다 성능 변화를 사전에 예측하고, 최적 설계에 활용할 수 있다.

\section{$\mathrm{IV}$. 결 론}

표적기가 레이다 포착 확률을 줄이고자 고기동을 통해 $\mathrm{RCS}$ 를 급변시킬 수 있고, 이는 전시 상황에서 레이다 탐 지성능에 지대한 영향을 미칠 수 있다. 공중전에서 표적 의 기동에 따른 $\mathrm{RCS}$ 변화는 레이다 성능 분석과 예측을 위해 반드시 확보해야 하지만, 실제 시험을 통해 $\mathrm{RCS}$ 를 측정하는 것은 시간, 비용이 많이 필요하기 때문에 $\mathrm{M} \& \mathrm{~S}$ 로 분석할 필요가 있다.

이에 따라 본 논문에서는 표적기 2종에 대해 기 확보한 1 도 간격의 전 방향 $\mathrm{RCS}$ 모의 데이터로부터 기동 시나리 오에 따른 레이다 성능을 모의 분석하고 검증하였다. 이 를 위해 시간자원을 고려한 레이다 모델과 표적 기동, 운 용환경 모델과 $\mathrm{RCS}$ 모의 데이터를 통합하여 $\mathrm{M} \& \mathrm{~S}$ 도구 를 개발하였다. 실측 수신신호 세기와 $\mathrm{RCS}$ 데이터를 적용 한 모의 결과를 비교함으로써 $\mathrm{RCS}$ 모의 데이터의 검증을 수행하고, 이동평균 방식을 적용함으로써 각도별 RCS 편 차를 줄인 $\mathrm{RCS}$ 가공 데이터의 적합성을 판단하였다.

이를 통해 표적의 고기동 상황에서 $\mathrm{RCS}$ 크기의 실시 간 변화로부터 레이다의 탐지 성능을 사전에 예측하고, 레이다 성능을 극대화할 수 있는 설계 최적화에 활용할 수 있을 것으로 기대된다.

\section{References}

[1] B. R. Mahafza, Radar Systems Analysis and Design using Matlab, 3rd ed. Boca Raton, FL, CRC Press, pp. 485490, 2013.

[2] M. A. Richards, Fundamentals of Radar Signal Processing, 2nd ed. New York, NY, McGraw-Hill, pp. 50-70, 2014.

[3] M. J. Lee, J. E. Kim, S. M. Lee, H. M. Jeon, W. Y. Yang, and K. T. Kim, "Detection of low-RCS targets in sea-clutter using multi-function radar," The Journal of Korean Institute of Electromagnetic Engineering and Science, vol. 30, no. 6, pp. 507-517, Jun. 2019.

[4] B. J. An, S. G. Seo, "A study on the setting procedure of standard value and design target value for the RCS reduced design for naval ships," The Journal of Korean Institute of Electromagnetic Engineering and Science, vol. 26, no. 6, pp. 581-588, Jun. 2015.

[5] J. J. Seo, "Analysis of tracking accuracy with consideration of fighter radar measurement characteristics," The Journal of Korean Institute of Electromagnetic Engineering and Science, vol. 29, no. 8, pp. 640-647, Aug. 2018.

[6] T. Y. Park, Y. R. Lee, "A study on radar received power based on target observing position," Journal of the Korea Institute of Information and Communication Engineering, vol. 18, no. 12, pp. 3063-3068, Dec. 2014.

[7] D. K. Barton, Radar System Analysis and Modeling, Norwood, MA, Artech House, 2005.

[8] W. L. Patterson, "Advanced refractive effects prediction system(AREPS)," in 2007 IEEE Radar Conference, Aug. 2007, pp. 891-895.

[9] R. S. Myong, T. H. Cho, "Development of a computational electromagnetics code for radar cross section calculations of flying vehhicles," Journal of the Korean Society for Aeronautical \& Space Sciences, vol. 33, no. 4, pp. 1-6, Apr. 2005.

[10] K. J. Kim, "A study on the analysis of radar cross section of aircraft and the measurement of radar cross section about scaled aircraft model," The Journal of the Korea Institute of Electronic Communication Sciences, vol. 15, no. 6, pp. 1055-1060, Dec. 2020.

[11] W. B. Kim, I. P. Hong, W. S. Oh, Y. J. Kim, and J. G. Yook, "Near to far field transformation algorithm for RCS analysis of large electrical objects," The Journal of Korean Institute of Electromagnetic Engineering and Science, vol. 30, no. 10, pp. 791-798, Oct. 2019. 
THE JOURNAL OF KOREAN INSTITUTE OF ELECTROMAGNETIC ENGINEERING AND SCIENCE. vol. 32, no. 5, May. 2021.

김 현 승 [LIG넥스원/선임연구원]

https: //orcid.org/0000-0003-4021-5914

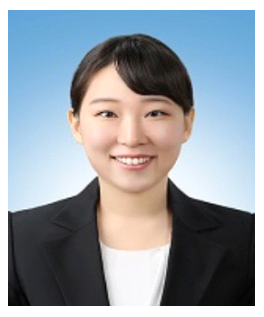

2014년 2월: 인하대학교 항공우주공학과 (공학사)

2016년 2월: 인하대학교 항공우주공학과 (공학석사)

2016년 1월 현재: LIG넥스원 SW연구소 선임연구원

[주 관심분야] 항공제어, $\mathrm{M} \& \mathrm{~S}$, 추적 알고 리즘 등

전 우 중 [LIG넥스원/선임연구원]

https: //orcid.org/0000-0003-3974-0631

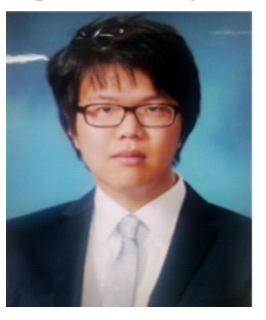

2010년 7월: 서울대학교 전기공학과 (공학 사)

2016년 2월: 서울대학교 전기공학과 (공학 석사)

2016년 1월 현재: LIG넥스원 SW연구소 선임연구원

[주 관심분야] $\mathrm{M \& S}$, 레이다, 제어 등
권 세 웅 [LIG넥스원/수석연구원]

https: //orcid.org/0000-0002-8959-4452

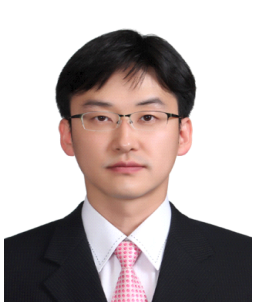

1999년 2월: 연세대학교 전파공학과 (공학

사)

2002년 2월: 연세대학교 전기컴퓨터공학 과 (공학석사)

2009년 8월: 연세대학교 전기전자공학과 (공학박사)

2009년 7월 현재: LIG넥스원 레이다연구 소 수석연구원

[주 관심분야] 능동위상배열레이다, 전파 전파 등

문 현 욱 [LIG넥스원/수석연구원]

https: //orcid.org/0000-0002-1298-2895

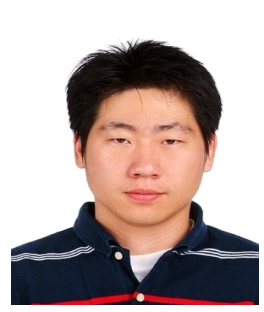

2005년 2월: 연세대학교 기계전자공학부 (공학사)

2007년 2월: 연세대학교 전기전자공학과 (공학석사)

2016년 2월: 연세대학교 전기전자공학과 (공학박사)

2014년 3월 현재: LIG넥스원 레이다연구 소 수석연구원

[주 관심분야] 레이다 성능분석, 전파 전파, 무선채널 등

박 명 훈 [LIG넥스원/선임연구원]

https://orcid.org/0000-0002-5217-0365

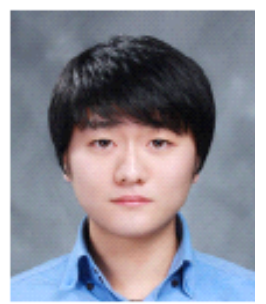

2014년 2월: 한국항공대학교 항공전자공 학과 (공학사)

2016년 2월: 한국항공대학교 항공전자정 보공학과 (공학석사)

2016년 1월 현재: LIG넥스원 SW연구소 선임연구원

안테나, 전파 전파 등

[주 관심분야] 레이다 체계 설계, $\mathrm{M} \& \mathrm{~S}$, 